\title{
Cardamonin as a potential treatment for melanoma induces human melanoma cell apoptosis
}

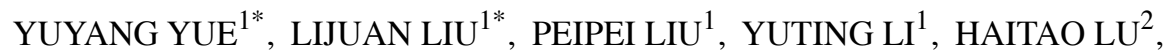 \\ YANJIA LI ${ }^{1}$, GUOQIANG ZHANG ${ }^{1}$ and XINSUO DUAN ${ }^{2}$ \\ ${ }^{1}$ Department of Dermatology, First Hospital, Hebei Medical University, Shijiazhuang, Hebei 050011; \\ ${ }^{2}$ Department of Dermatology, The Affiliated Hospital of Chengde Medical College, Chengde, Hebei 067000, P.R. China
}

Received November 17, 2018; Accepted October 22, 2019

DOI: $10.3892 / 01.2019 .11242$

\begin{abstract}
. 2',4'-dihydroxy-6'-methoxychalcone (cardamonin) is a natural compound with anti-proliferative effects on several cancer types including nasopharyngeal carcinoma. The effects of cardamonin on melanoma cells are unknown. The present study investigated the anti-proliferative effect of cardamonin on human melanoma cell lines (M14 and A375), and the underlying apoptosis inducing mechanisms. MTS assay showed that cardamonin inhibited M14 cells viability, and a reduction of the M14 cell density was also observed. Flow cytometry showed that cardamonin induced M14 cells apoptosis in a dose-dependent manner. Western blot analysis showed protein expression in M14 and A375; the pro-apoptotic protein BAX was upregulated, while the anti-apoptotic protein B-cell lymphoma-2 was downregulated. The protein expression of cleaved caspase-8, -9 and cleaved poly (ADP-ribose) polymerase was increased, whereas P65 was decreased. Furthermore, cardamonin inhibited M14 cell migration. These findings suggest that cardamonin may be a novel anticancer treatment for human melanoma.
\end{abstract}

\section{Introduction}

Human melanoma is a highly malignant tumor derived from abnormal melanocytes. It is responsible for $>75 \%$ of skin cancer deaths (1). Melanomas metastasize in the lymph nodes through blood vessels and the prognosis is poor. Melanoma treatment by known drugs and therapies is limited

Correspondence to: Dr Guoqiang Zhang, Department of Dermatology, First Hospital, Hebei Medical University, 89 Donggang Road, Shijiazhuang, Hebei 050011, P.R. China E-mail: zlx090702@163.com

Dr Xinsuo Duan, Department of Dermatology, The Affiliated Hospital of Chengde Medical College, Chengde, Hebei 067000, P.R. China

E-mail: duanxinsuo2002@163.com

Key words: cardamonin, melanoma, apoptosis, extrinsic pathway, extrinsic pathway and outcome is poor. The incidence of a five-year survival rate ranges between $5-15 \%$ (2). Although the effectiveness of dacarbazine as a single-agent therapy is only $4.5 \%$, it is commonly used for the treatment of advanced melanoma as a chemotherapeutic agent (3). Moreover the majority of patients using a mono- or combination therapy comprising single-agent BRAF and/or dual specificity mitogen-activated protein kinase kinase (MEK) kinase inhibitors only exhibited a clinical improvement for a limited time (4). Therefore, the discovery of new treatments is important for the short-term prognosis of patients with melanoma.

Natural products are a potential source for new therapies. More than $75 \%$ of new anti-tumor drugs have originated from natural products, for example, paclitaxel and colchicine (5). The Food and Drug Administration (FDA) has approved $25-48 \%$ of plant derived medicines (6).

2 ',4'-dihydroxy-6'-methoxychalcone (cardamonin) is a natural chalcone compound that is extracted from members of the Zingiberaceae family (7). It may increase cell apoptosis in several tumors, including nasopharyngeal carcinoma, prostate cancer and glioblastoma (8-10). Cardamomin may be effective via multiple signaling pathways, including nuclear factor $(\mathrm{NF})-\kappa \mathrm{B}$, signal transducer and activator of transcription 3 and mammalian target of rapamycin pathways $(8,10,11)$. However, the underlying effects and mechanisms of cardamonin on melanoma cells remain unclear. In the present study, several assays were used to determine the anticancer activity and effects of cardamonin on human melanoma cells.

\section{Materials and methods}

Cell viability assay. An MTS assay was used to determine the effect of cardamonin on melanoma cell viability. Cells were cultured in RPMI-1640 Medium (Corning, cat. no. 10-040-CV) with 10\% FBS (Shanghai ExCell Biology, Inc.; cat. no. FND500) in an incubator $\left(37^{\circ} \mathrm{C}, 50 \% \mathrm{CO}_{2}\right)$ Human melanoma M14 cells, supplied by the Chinese Academy of Medical Sciences, $\left(2-5 \times 10^{3} / 100 \mu \mathrm{l} /\right.$ well $)$ were seeded in a 96-well plate and incubated for $24 \mathrm{~h}$ pre-cardamonin treatment at different concentrations $(0,30,60$ and $90 \mu \mathrm{M})$ for $24 \mathrm{~h}$. Thereafter, $20 \mu \mathrm{l}$ MTS solution $(2 \mathrm{mg} / \mathrm{ml})$ was added to each well followed by a $2 \mathrm{~h}$ incubation period. Absorbance 
was measured with a microplate reader (Infinite F50; Tecan Schweiz AG) at a wavelength of $495 \mathrm{~nm}$.

Flow cytometry. Cell apoptosis caused by cardamonin was measured with a fluorescein isothiocyanate (FITC) Annexin V apoptosis detection kit (BD Biosciences; cat. no. 556547). M14 cells were seeded in a 6-well plate and treated with varying cardamonin concentrations $(0,30,60$ and $90 \mu \mathrm{M})$ for 24 h. Cells were collected and washed with ice-cold PBS and incubated with Annexin V-FITC and propidium iodide (PI) in the dark for $20 \mathrm{~min}$. The cells were then resuspended with binding buffer and measured using a Navios flow cytometer B47903 and Navios Platform System Software v2.0 (both Beckman Coulter, Inc.).

Western blot analysis. M14 and A375 cell lines (Chinese Academy of Medical Science) were seeded in 6-well plates $\left(2 \times 10^{5}\right.$ per well $)$ and treated with varying cardamonin concentrations $(0,30,60$ and $90 \mu \mathrm{M})$ for $24 \mathrm{~h}$. Cell proteins were collected with RIPA buffer (Beijing Solarbio Science \& Technology Co., Ltd.; cat. no. R0020-100 ml) supplemented with PMSF. Protein concentrations were measured using a bicinchoninic acid protein assay kit (MultiSciences). The proteins $(30 \mu \mathrm{g})$ were separated via SDS-PAGE (15\% gel) electrophoresis and transferred to PVDF membranes. The membranes were then blocked in 5\% non-fat milk in TBST (0.05\%Tween 20$)$ for $1 \mathrm{~h}$ at room temperature and incubated with homologous primary antibodies (all 1:1,000) overnight at $4^{\circ} \mathrm{C}[\beta$-actin, cat. no. AC026; P65, cat. no. A2547; BCL2, cat. no. A2212; BAX, cat. no. A7626 (all ABclonal Biotech Co., Ltd.); Cleaved Caspase-8, cat. no. 9496; Cleaved Caspase-9, cat. no. 20750; Cleaved PARP, cat. no. 5625 (all Cell Signaling Technology, Inc.)]. The membranes were incubated with horse radish peroxidase conjugated secondary antibodies (Goat Anti-Rabbit IgG horseradish-peroxidase conjugated; cat. no. ab205718; 1:10,000) at room temperature for $2 \mathrm{~h}$. After washing the membranes, enhanced chemiluminescence reagents (Beijing Solarbio Science \& Technology Co., Ltd.; cat. no. PE0010) were applied to them before being scanned by a ChemiDoc XRS+ system (Bio-Rad Laboratories, Inc.).

Wound healing assay. A wound healing assay was used to test the effect of cardamonin on cell migration. M14 cells were seeded into 6-well plates $\left(5 \times 10^{5}\right.$ per well) (Guangzhou Jet Bio-Filtration Co., Ltd.; cat. no. TCP010006) and cultured to $80 \%$ confluence. They were then scratched through the cell monolayer using a $10 \mu \mathrm{l}$ pipette tip before being washed with serum-free RPMI-1640 medium (Corning, Inc., cat. no. 15-040-CV) to remove floating cells. Subsequently, cells were treated with varying cardamonin concentrations $(0,30,60$ and $90 \mu \mathrm{M})$ for $24 \mathrm{~h}$ and images were captured using the ChemiDoc ${ }^{\mathrm{TM}}$ XRS+ System light miscroscope with Image Lab $^{\text {TM }}$ Software (Bio-Rad Laboratories, Inc., cat. no. 1708265).

Data analysis. The Gray value of three repeats was measured using ImageJ (1.50i; National Institutes of Health). Data are presented as the mean \pm standard deviation. The significance difference between distinct groups were determined using one-way ANOVA (SPSS 21.0; IBM Corp.) with post-hoc test

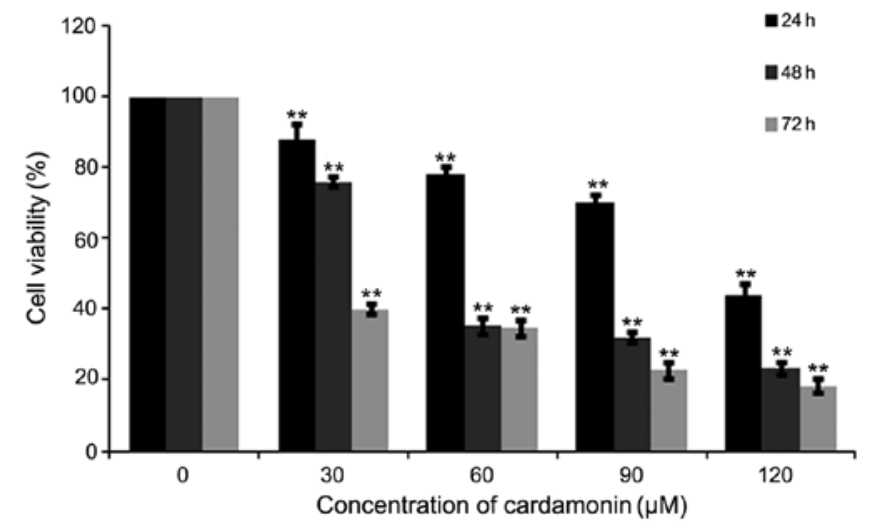

Figure 1. Effects of cardimonin on M14 cells growth. M14 cells were treated with various concentrations of cardamonin $(0,30,60,90$ and $120 \mu \mathrm{M})$ for 24 , 48 and 72 h. Cell viability was measured using an MTS assay. Graphs represent average percentages of cell proliferation normalized to M14 cells in the control group. Significance levels between distinct groups were determined using one-way analysis of variance. ${ }^{* *} \mathrm{P} \leq 0.01$ vs. control.

(Fisher's Least Significant Difference). $\mathrm{P}<0.05$ was considered to indicate a statistically significant difference.

\section{Results}

Cardamonin inhibits cell viability. M14 cells were treated with varying cardamonin concentrations $(0,30,60,90$ and $120 \mu \mathrm{M} ; \mathrm{n}=4)$ for 24,48 and $72 \mathrm{~h}$. The MTS assay showed that cardamonin significantly decreased M14 cell viability $(\mathrm{P}<0.01$; Fig. 1). Cell density was also decreased after cardamonin treatment for $24 \mathrm{~h}$ (Fig. 2). The results indicated that cardamonin could inhibit M14 cell growth.

Cardamonin induces apoptosis in M14 cells. M14 cells were cultured in varying cardamonin concentrations $(0,30,60$ and $90 \mu \mathrm{M}, \mathrm{n}=3$ ) for $24 \mathrm{~h}$ and then stained with Annexin V-FITC and PI for flow cytometry analysis. Results indicated that the untreated M14 cells were not stained by Annexin V and PI, whereas a significant dose-dependent increase was noted in the Annexin V and PI positive cells following cardamonin treatment $(\mathrm{P}<0.01$; Fig. 3$)$.

Cardamonin alters apoptosis-associated proteins in M14 cells and A375 cells. In order to investigate whether cardamonin induced melanoma cell apoptosis via the effects of apoptosis-associated proteins, immunoblotting was performed. Protein lysates were collected from two different cell lines (M14 and A375). They were cultured in varying cardamonin concentrations $(0,30,60$ and $90 \mu \mathrm{M} ; \mathrm{n}=3)$ for $24 \mathrm{~h}$. It was found that the pro-apoptotic protein BAX was upregulated, while the anti-apoptotic protein B-cell lymphoma-2 (BCL2) was downregulated in both cell lines. Intrinsic or extrinsic pathways may be involved in apoptosis, therefore the expression of cleaved caspase-8, -9 and cleaved PARP were investigated (12). These apoptotic signaling proteins increased significantly after 24-h treatment with varying cardamonin concentrations $(\mathrm{P}<0.01)$. P65 expression significantly decreased, thus indicating that cardamonin is a major anti-apoptotic regulator $(\mathrm{P}<0.01$; Figs. 4 and 5). Results showed that cardamonin regulates 

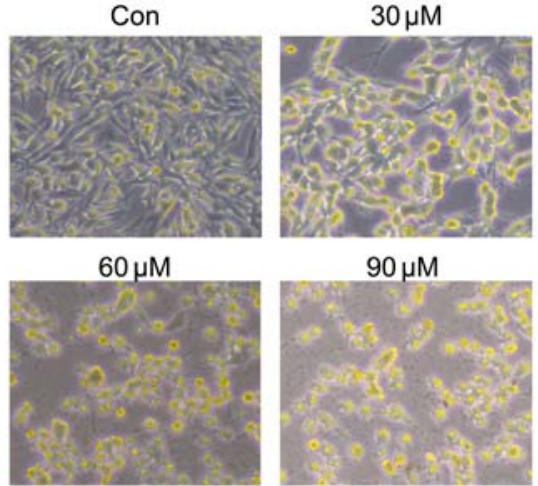

$90 \mu \mathrm{M}$

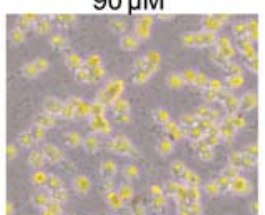

Figure 2. Effects of cardamonin on M14 cell density. M14 cells were treated with various concentration of cardamonin $(0,30,60$ and $90 \mu \mathrm{M})$ for $48 \mathrm{~h}$. Images are representative of three independent experiments. Con, control.
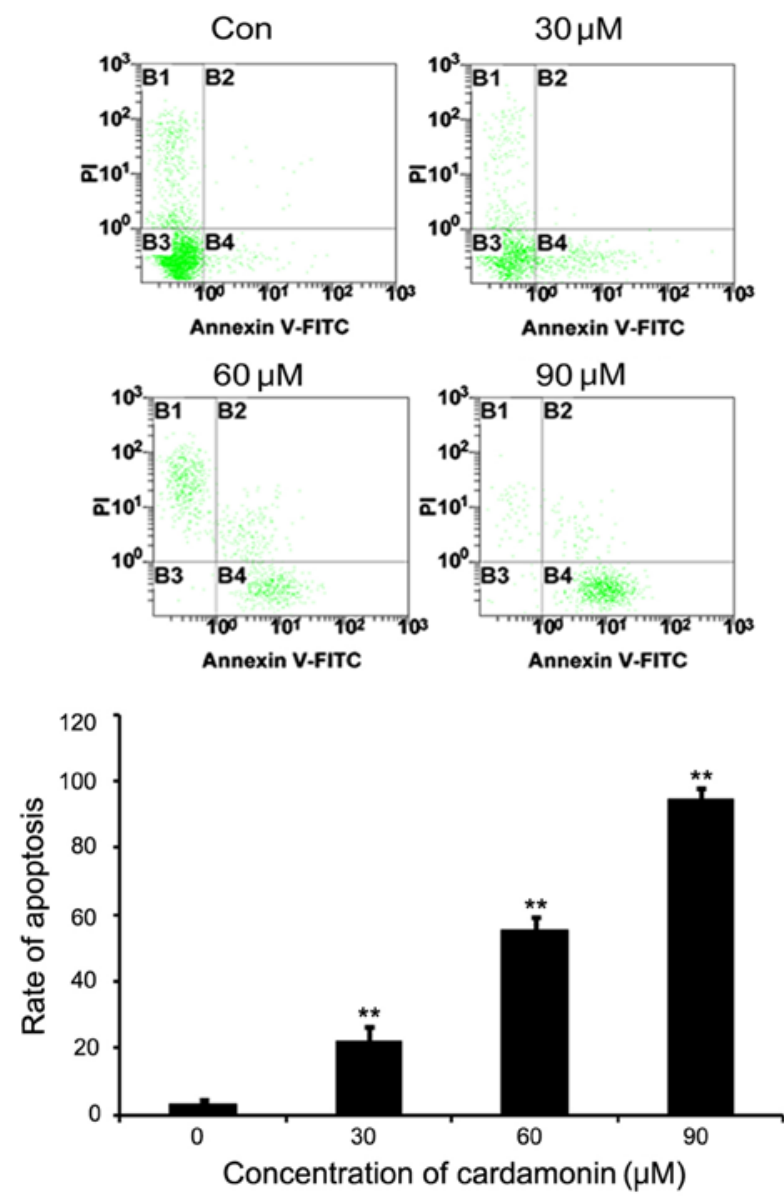

Figure 3. Effects of cardamonin on M14 cell apoptosis. M14 cells were treated with different concentrations of cardamonin $(0,30,60$ and $90 \mu \mathrm{M})$ for $24 \mathrm{~h}$. FITC, fluorescein isothiocyanate; PI, propidium iodide; Con, control. ${ }^{* *} \mathrm{P} \leq 0.01$ vs. control.

the expression of important pro-apoptotic and anti-apoptotic proteins.

Cardamonin suppresses M14 cell migration. To assess whether cardmonin affects cell migration, a scratch-wounding assay was performed on M14 cells. Under varying cardamonin concentrations $(0,30,60$ and $90 \mu \mathrm{M} ; \mathrm{n}=3)$, the open wound areas were significantly increased in comparison to the untreated cells
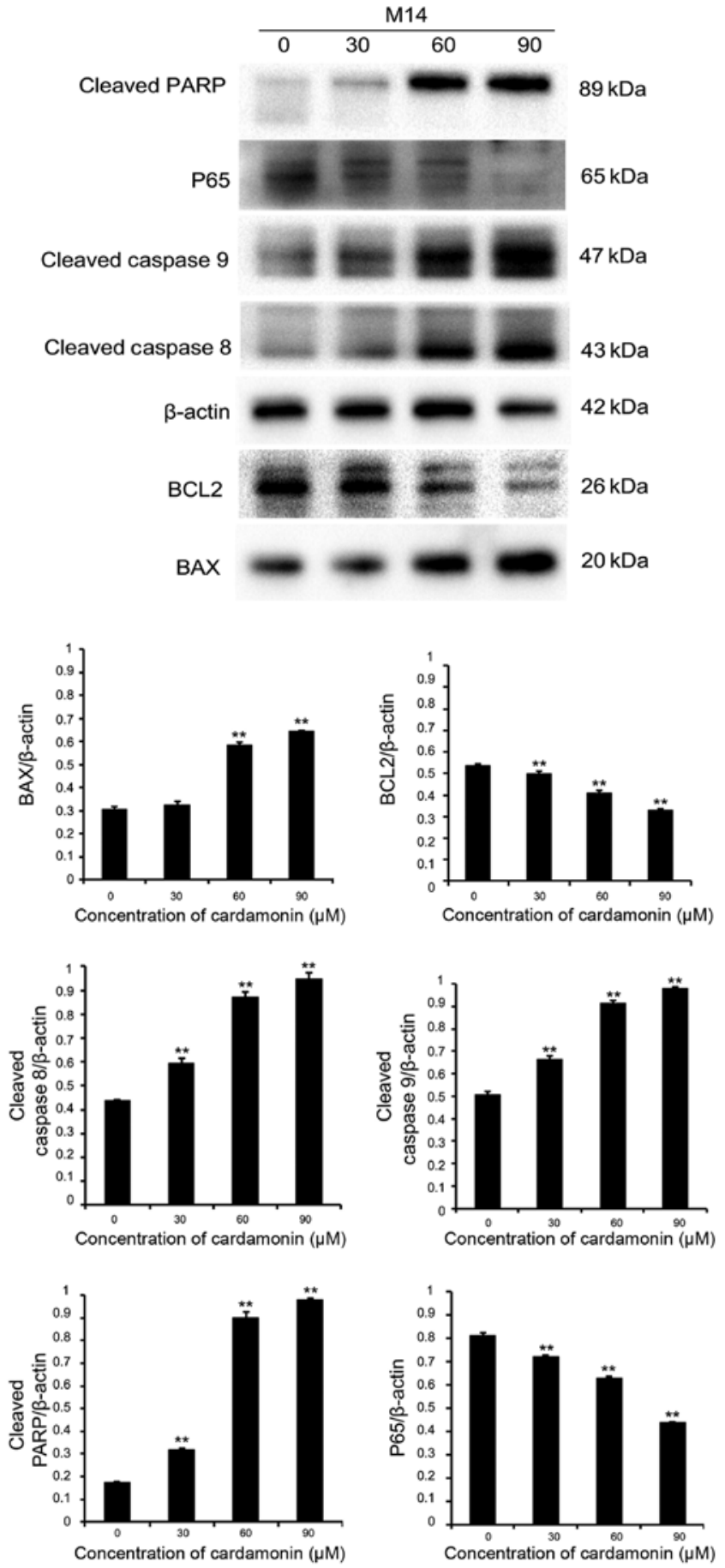

Figure 4. Protein expression of Bax, BCL2, cleaved caspase-8, cleaved caspase- 9 , cleaved PARP, nuclear factor- $\kappa \mathrm{B}$ p65 in M14 cell line detected by western blot analysis. $\beta$-actin was used as an internal control. The levels of different proteins were quantified and normalized to $\beta$-actin and are shown in a histogram. Significance levels between distinct groups were determined using one-way analysis of variance. ${ }^{* *} \mathrm{P} \leq 0.01$ vs. the control. PARP, poly (ADP-ribose) polymerase; BCL2, B-cell lymphoma-2.

$(\mathrm{P}<0.01)$. These data indicated that cardamonin suppressed M14 cell migration in a dose-dependent manner (Fig. 6).

\section{Discussion}

Cutaneous melanoma is responsible for $1.6 \%$ of new cancer cases and $0.7 \%$ of cancer-related deaths worldwide (13). 

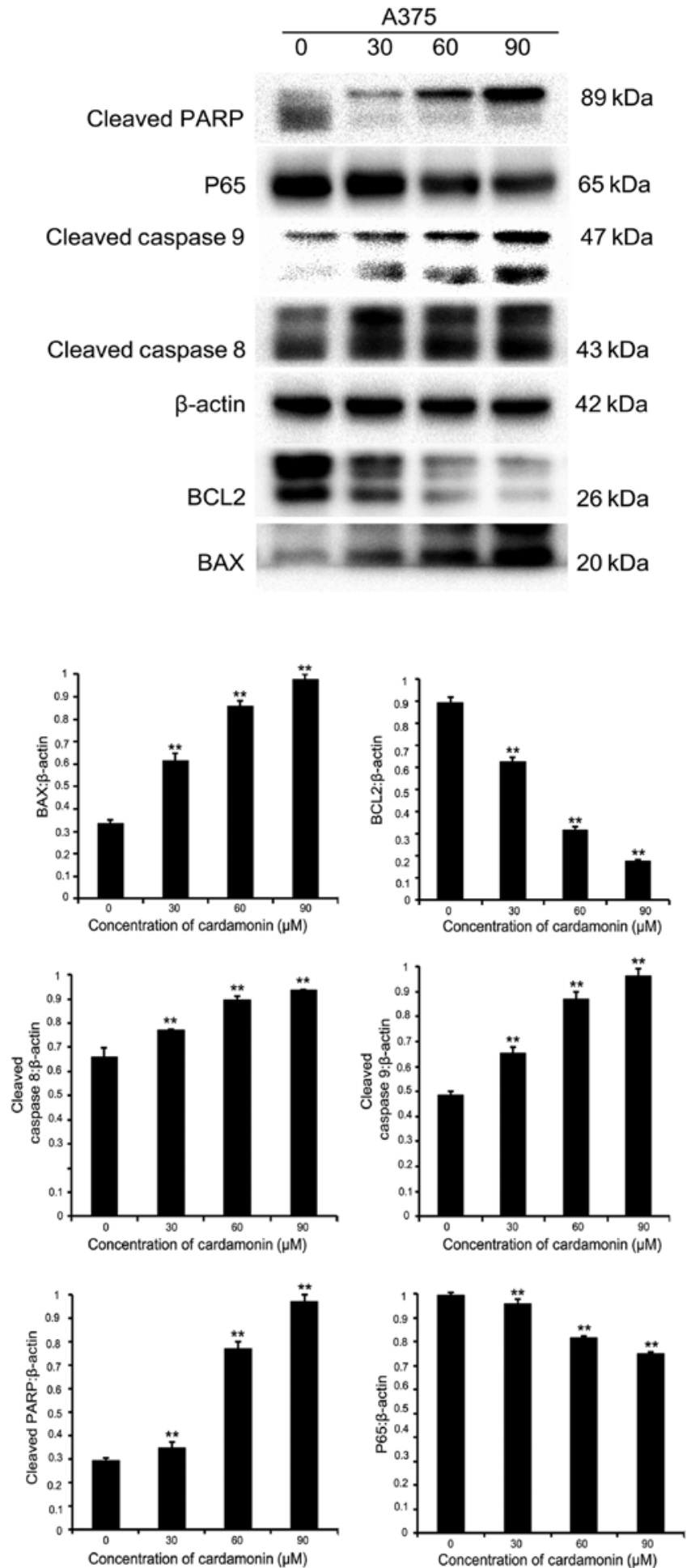

Figure 5. Protein expression of Bax, BCL2, cleaved caspase-8, cleaved caspase-9, cleaved PARP, NF- $\mathrm{B}$ p65 in A375 cell line is detected by western blot analysis. $\beta$-actin was used as an internal control. Significance levels between distinct groups were determined using one-way analysis of variance. ${ }^{* *} \mathrm{P} \leq 0.01$ vs. the control. PARP, poly (ADP-ribose) polymerase; BCL2, B-cell lymphoma-2.

Traditional chemotherapy is ineffective in melanoma treatment and the mortality rate remains high. Dakabazine (DTIC) is the only FDA-approved anti-melanoma chemotherapy drug in use to date. In comparison to supportive therapies (5-11 months), the response rate to DTIC ranges from between

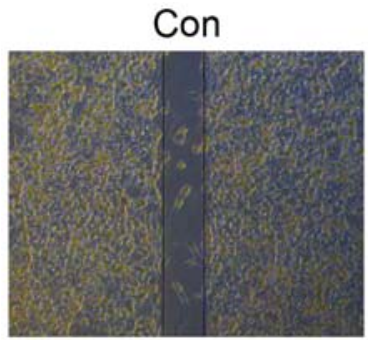

$60 \mu \mathrm{M}$
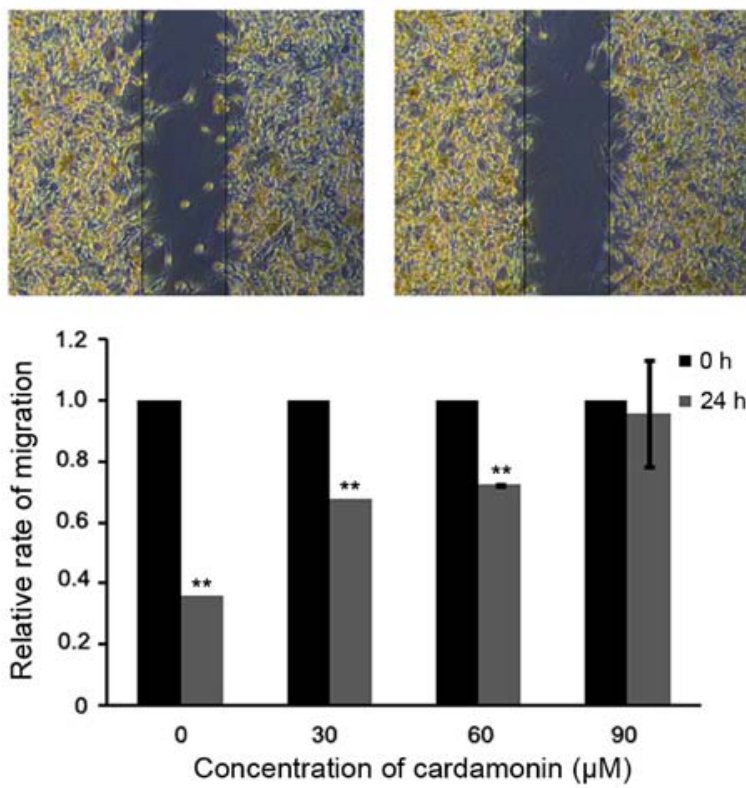

Figure 6. M14 cells are treated with different concentrations of cardamonin $(0,30,60$ and $90 \mu \mathrm{M})$ for $24 \mathrm{~h}$, and migration is inhibited. The levels of different groups were quantified and are shown in a histogram. Significance levels between distinct groups were measured and analyzed using one-way analysis of variance. ${ }^{* *} \mathrm{P} \leq 0.01$ vs. control.

$5-25 \%$ and does not increase overall survival. The development of targeted therapy has improved the response and overall survival rate in melanoma patients. Vemurafenib as an orally active BRAF inhibitor, exhibits partial and complete responses in BRAF mutated melanoma patients. Melanoma cells may easily develop drug resistance (14). Dabrafenib as another BRAF inhibitor that is similar to vemurafenib in terms of progression-free survival (PFS) and reaction rate (15). Approved immunotherapeutics include ipilimumab and nivolumab, the former being a monoclonal antibody that blocks the cytotoxic T-lymphocyte protein-4 receptor. Previous studies have shown that the combination of nivolumab (a PD-1 inhibitor) may achieve better results than the single use of ipilimumab (16-18). Nivolumab monotherapy has a higher objective response rate, a longer PFS and overall survival rate compared to chemotherapy treatments. Despite the positive effects of immunotherapy drugs, a large number of patients continue to succumb to melanoma metastasis. The ubiquitous resistance to molecular targeted therapy remains a challenge for the treatment of melanoma.

Therefore, the effective treatment of melanoma depends on the development of novel medicines. Furthermore, researchers are committed to finding drugs that are effective on cancer cells while having minimal cytotoxic effects on normal cells. 


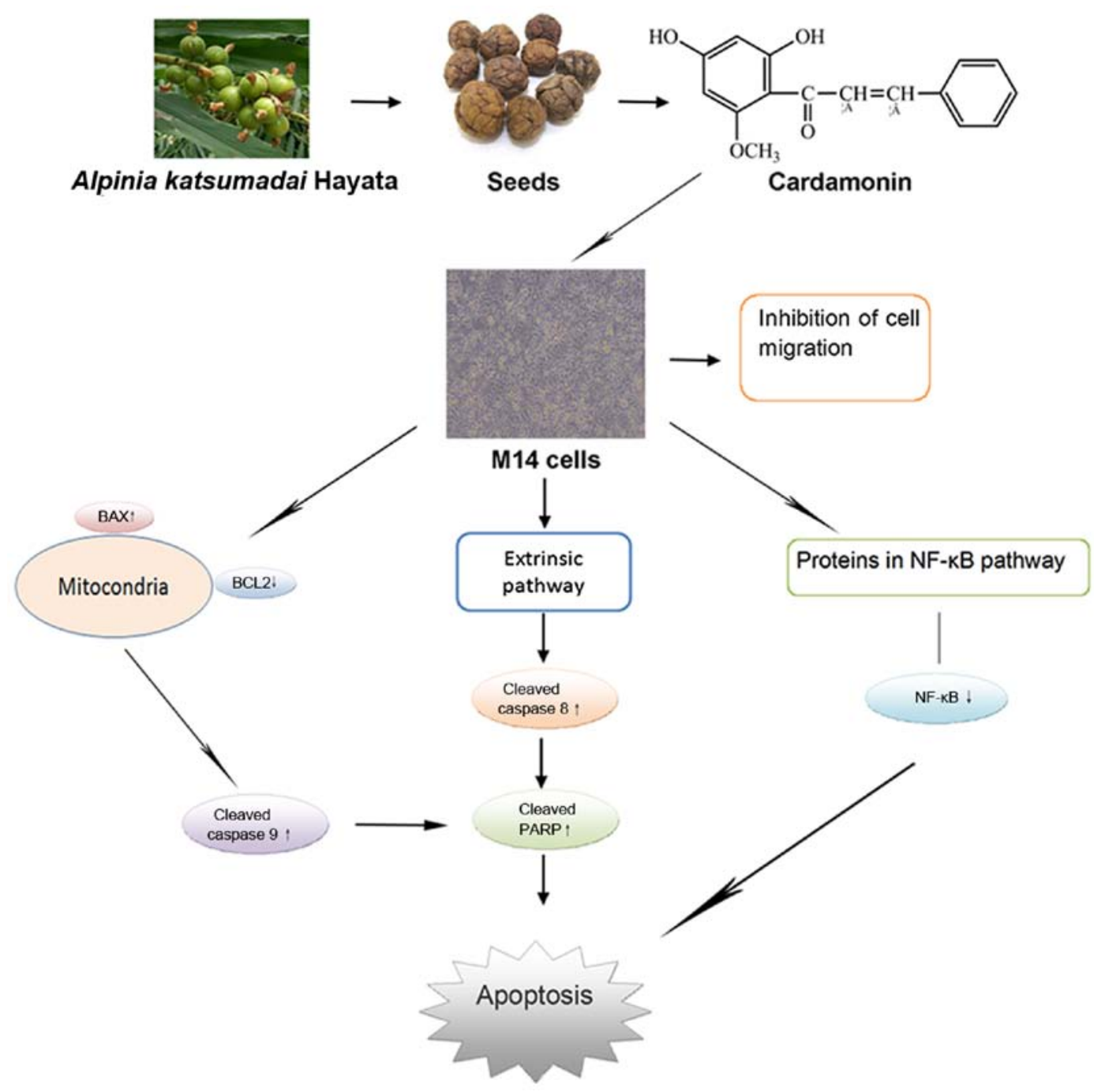

Figure 7. Flow diagram. Cardamonin, a natural chalcone compound, can induce melanoma cell apoptosis and change the expression of apoptosis-associated proteins. PARP, poly (ADP-ribose) polymerase; BCL2, B-cell lymphoma-2.

Alpinia katsumadai hayata is a traditional Chinese herbal medicine. It induces a warming of the stomach and is used for relieving gastric discomfort and a distended abdomen (19). Cardamonin is extracted from the seed of cardamom spices and it is an active ingredient of Alpinia katsumadai hayata, which has antinociceptive effects $(15,20)$. Cardamonin has been tested as an anticancer treatment in several tumors, except melanoma. Cardamonin's proven safety and effects in tumor treatment make it a promising anti-cancer reagent. An MTS assay was performed to verify its anti-proliferative effect on M14 melanoma cells.

Apoptosis is an important physiological process whereby cells commit suicide (21). There are two major pathways of apoptosis, namely the intrinsic and the extrinsic pathway. The intrinsic pathway is controlled by the BCL2 family of proteins, which may be classified as pro-apoptotic proteins like BAX and anti-apoptotic proteins such as BCL2. BAX as a factor in the BCL2 family, plays an important role in cell apoptosis and mitochondrial function (22). BCL2 may induce apoptosis by binding to anti-apoptotic proteins, thereby replacing activators that may activate BAX (23). BCL2 is a fundamental anti-apoptotic gene that that is recognized in cancer development (24).
Overexpression of BCL2 promotes tumorigenesis and tumor progression and is associated with poor patient prognosis in numerous types of cancer, for example, breast cancer, prostate cancer and melanoma (25). In order to achieve more reliable results, both M14 and A375 cell were treated with cardamonin and analyzed by western blotting. It was noted that treatment with cardamonin resulted in the downregulation of BCL2 and upregulation of BAX protein levels in M14 and A375 cells. Both proteins play a pivotal role in apoptotic pathway regulation.

Caspases are also vital pro-apoptotic proteins. Caspase- 8 occurs in extrinsic apoptotic pathways while caspase- 9 is involved in intrinsic (mitochondrial) pathways. Caspase- 8 can process classical apoptotic caspases including caspase- 9 , which may lead to apoptotic initiation (26). Proenzymes synthesized by caspases, are activated by cleaving the pro-domain at a specific aspartic acid cleaving site. Activation of caspase- 8 or -9 may eventually lead to the cleavage of poly (ADP-ribose) polymerase (PARP) which results in DNA fragmentation and apoptosis (27). In a previous study, upregulation of cleavage of caspase- 8 , caspase- 9 and PARP was investigated in the treatment of prostate cancer cells with cardamonin (9). Western blot 
analysis results showed apoptotic induction by cardamonin via the cleavage of caspase- $8,-9$ and PARP. These results reflected those of a previous study that performed a Caspase-Glo 3/7, 8 and 9 assay and western blot analysis in A549 and HK1 cells (28). Ma et al (29) discovered that Deoxyarbutin inhibited Bcl-2, activated Bax and voltage-dependent anion-selective channel protein, which in turn sequentially activated caspase-9, PARP, caspase- 3 and finally led to mitochondria associated apoptosis. While in a study by Bush et al (30) it was revealed that another drug curcumin induced melanoma cell death by activating caspases- 3 and caspases- 8 but not caspase- 9 . Therefore, the role of caspase- 9 in melanoma cells needs to be further investigated.

NF- $\kappa$ B (P65) which combines with fixed nucleotide sequences of various gene promoters, is an anti-apoptotic factor. P65 has an important influence on cell proliferation and differentiation, tumor formation, invasion and metastasis. P65 directly mediates vital tumor-promoting mechanisms. NF- $\kappa \mathrm{B}$ is known to activate cell proliferation, prevent apoptosis, promote tumor angiogenesis, epithelial-to-mesenchymal transition, invasiveness and metastasis (31). P65 phosphorylation is associated to the expression of the pro-apoptotic BCL2 family, which includes Bcl-2 (32). A study by Wang and Liu (33) confirmed that ingenol-3-angelate suppressed the growth of melanoma cells, in which P65 activity was downregulated. Furthermore, the effects of cardamonin on cancer cell apoptosis via the P65 signaling pathway have been proven. P65 expression was tested in M14 and A375 cell lines exposed to cardamonin by means of western blot analysis in the present study. Cardamonin did not inhibit P65 in these cell lines.

The present study demonstrated that cardamonin promotes apoptosis of melanoma cells via the regulation of proteins in the intrinsic, extrinsic and NF- $\kappa$ B pathways (Fig. 7). Cardamonin, as a natural compound, may be a potentially valuable factor for human melanoma treatment.

\section{Acknowledgements}

Not applicable.

\section{Funding}

Financial assistance was provided by the Hebei Provincial Administration of Traditional Chinese Medicine (project number: 2018055).

\section{Availability of data and materials}

The datasets used and/or analyzed during the current study are available from the corresponding author on reasonable request.

\section{Authors' contributions}

YY, LL, YTL, PPL and YJL analyzed and collected the data regarding the MTS and the western blotting analysis. HL analyzed and collected the data regarding the flow cytometry analysis. GZ and XD helped with experimental design, interpretation of data and made major contributions to the writing of the manuscript. All authors read and approved the final manuscript.

\section{Ethics approval and consent to participate}

Not applicable.

\section{Patient consent for publication}

Not applicable.

\section{Competing interests}

The authors declare that they have no competing interests.

\section{References}

1. Lee C, Collichio F, Ollila D and Moschos S: Historical review of melanoma treatment and outcomes. Clin Dermatol 31: 141-147, 2013.

2. Chien AJ, Moore EC, Lonsdorf AS, Kulikauskas RM, Rothberg BG, Berger AJ, Major MB, Hwang ST, Rimm DL and Moon RT: Activated Wnt/beta-catenin signaling in melanoma is associated with decreased proliferation in patient tumors and a murine melanoma model. Proc Natl Acad Sci USA 106: 1193-1198, 2009.

3. Cui C, Mao L, Chi Z, Si L, Sheng X, Kong Y, Li S, Lian B, $\mathrm{Gu} \mathrm{K}$, Tao M, et al: A phase II, randomized, double-blind, placebo-controlled multicenter trial of Endostar in patients with metastatic melanoma. Mol Ther 21: 1456-1463, 2013.

4. Sullivan R, LoRusso P, Boerner S and Dummer R: Achievements and challenges of molecular targeted therapy in melanoma. Am Soc Clin Oncol Educ Book 2015: 177-186.

5. Atanasov AG, Waltenberger B, Pferschy-Wenzig EM, Linder T, Wawrosch C, Uhrin P, Temml V, Wang L, Schwaiger S, Heiss EH, et al: Discovery and resupply of pharmacologically active plant-derived natural products: A review. Biotechnol Adv 33: 1582-1614, 2015.

6. Harvey AL, Edrada-Ebel R and Quinn RJ: The re-emergence of natural products for drug discovery in the genomics era. Nat Rev Drug discov 14: 111-129, 2015.

7. Goncalves LM, Valente IM and Rodrigues JA: An overview on cardamonin. J Med Food 17: 633-640, 2014.

8. Li Y, Qin Y, Yang C, Zhang H, Li Y, Wu B, Huang J, Zhou X, Huang B, Yang K and Wu G: Cardamonin induces ROS-mediated $\mathrm{G} 2 / \mathrm{M}$ phase arrest and apoptosis through inhibition of NF- $\kappa \mathrm{B}$ pathway in nasopharyngeal carcinoma. Cell Death Dis 8: e3024, 2017.

9. Zhang J, Sikka S, Siveen KS, Lee JH, Um JY, Kumar AP, Chinnathambi A, Alharbi SA, Basappa, Rangappa KS, et al: Cardamonin represses proliferation, invasion, and causes apoptosis through the modulation of signal transducer and activator of transcription 3 pathway in prostate cancer. Apoptosis 22: 158-168, 2017.

10. Wu N, Liu J, Zhao X, Yan Z, Jiang B, Wang L, Cao S, Shi D and Lin X: Cardamonin induces apoptosis by suppressing STAT3 signaling pathway in glioblastoma stem cells. Tumour Biol 36: 9667-9676, 2015

11. Niu P, Shi D, Zhang S, Zhu Y and Zhou J: Cardamonin enhances the anti-proliferative effect of cisplatin on ovarian cancer. Oncol Lett 15: 3991-3997, 2018.

12. Elmore S: Apoptosis: A review of programmed cell death Toxicol Pathol 35: 495-516, 2007.

13. Ferlay J, Soerjomataram I, Dikshit R, Eser S, Mathers C, Rebelo M, Parkin DM, Forman D and Bray F: Cancer incidence and mortality worldwide: Sources, methods and major patterns in GLOBOCAN 2012. Int J Cancer 136: E359-E386, 2015.

14. Rosen LS, Lorusso P, MA WW, Goldman JW, Weise A, Colevas AD, Adjei A, Yazji S, Shen A, Johnston S, et al: A first-in-human phase I study to evaluate the MEK1/2 inhibitor, cobimetinib, administered daily in patients with advanced solid tumors. Invest New Drugs 34: 604-613, 2016.

15. Park MK, Lee HJ, Choi JK, Kim HJ, Kang JH, Lee EJ, Kim YR, Kang JH, Yoo JK, Cho HY, et al: Novel anti-nociceptive effects of cardamonin via blocking expression of cyclooxygenase-2 and transglutaminase-2. Pharmacol Biochemistry Behav 118: 10-15, 2014. 
16. Larkin J, Chiarion-Sileni V, Gonzalez R, Grob JJ, Cowey CL, Lao CD, Schadendorf D, Dummer R, Smylie M, Rutkowski P, et al: Combined nivolumab and ipilimumab or monotherapy in untreated melanoma. N Engl J Med 373: 23-34, 2015.

17. Caroline R, Jacob S, Georgina VL, Ana A, Jean JG, Laurent M, Adil D, Matteo SC, Catriona M, Michal L, et al: Pembrolizumab versus ipilimumab in advanced melanoma. N Engl J Med 372: 2521-2532, 2015

18. Caroline R, Georgina VL, Jacob S, Ana A, Jean JG, Laurent M, Adil D, Matteo SC, Catriona M, Michal L, et al: Long-term outcomes in patients (pts) with ipilimumab (ipi)-naïve advanced melanoma in the phase 3 KEYNOTE-006 study who completed pembrolizumab (pembro) treatment. J Clin Oncol 15 (Suppl) S35-S35, 2017.

19. Wang S, Zhai C, Zhang Y, Yu Y, Zhang Y, Ma L, Li S and Qiao Y: Cardamonin, a novel antagonist of hTRPA1 cation channel, reveals therapeutic mechanism of pathological pain. Molecules 21: E1145, 2016.

20. Liu L, Chen X and Hu Z: Separation and determination of alpinetin and cardamonin in alpinia katsumadai hayata by flow injection-micellar electrokinetic chromatography. Talanta 71 : 155-159, 2007.

21. Fulda S, Gorman AM, Hori O and Samali A: Cellular stress responses: Cell survival and cell death. Int J Cell Biol 2010 214074, 2010.

22. Chan SL and Yu VC: Proteins of the bcl-2 family in apoptosis signalling: From mechanistic insights to therapeutic opportunities. Clin Exp Pharmacol Physiol 31: $119-128,2004$

23. Hata AN, Engelman JA and Faber AC: The BCL2 family: Key mediators of the apoptotic response to targeted anticancer therapeutics. Cancer Discov 5: 475-487, 2015.

24. Zhu Y, Wen X and Zhao P: MicroRNA-365 inhibits cell growth and promotes apoptosis in melanoma by targeting BCL2 and cyclin D1 (CCND1). Med Sci Monit 24: 3679-3692, 2018.
25. Radha G and Raghavan SC: BCL2: A promising cancer therapeutic target. Biochim Biophys Acta Rev Cancer 1868: 309-314, 2017.

26. Monie TP and Bryant CE: Caspase- 8 functions as a key mediator of inflammation and pro-IL-1 $\beta$ processing via both canonical and non-canonical pathways. Immunol Rev 265: 181-193, 2015.

27. Li J and Yuan J: Caspases in apoptosis and beyond. Oncogene 27 6194-6206, 2008.

28. Break MKB, Hossan MS, Khoo Y, Qazzaz ME, Al-Hayali MZK, Chow SC, Wiart C, Bradshaw TD, Collins H and Khoo TJ Discovery of a highly active anticancer analogue of cardamonin that acts as an inducer of caspase-dependent apoptosis and modulator of the mTOR pathway. Fitoterapia 125: 161-173, 2018.

29. Ma L, Xu Y, Wei Z, Xin G, Xing Z, Niu H and Huang W: Deoxyarbutin displays antitumour activity against melanoma in vitro and in vivo through a p38-mediated mitochondria associated apoptotic pathway. Sci Rep 7: 7197, 2017.

30. Bush JA, Cheung KJ Jr and LI G: Curcumin induces apoptosis in human melanoma cells through a Fas receptor/caspase-8 pathway independent of p53. Exp Cell Res 271: 305-314, 2001.

31. Kaltschmidt B, Greiner JFW, Kadhim HM and Kaltschmidt C: Subunit-specific role of NF- $\kappa \mathrm{B}$ in cancer. Biomedicines 6: E44, 2018.

32. Turillazzi E, Neri M, Cerretani D, Cantatore S, Frati P, Moltoni L, Busardò FP, Pomara C, Riezzo I and Fineschi V: Lipid peroxidation and apoptotic response in rat brain areas induced by long-term administration of nandrolone: The mutual crosstalk between ROS and NF-kB. J Cell Mol Med 20: 601-612, 2016.

33. Wang D and Liu P: Ingenol-3-Angelate suppresses growth of melanoma cells and skin tumor development by downregulation of NF-кB-cox2 signaling. Med Sci Monit 24: 486-502, 2018.

This work is licensed under a Creative Commons Attribution-NonCommercial-NoDerivatives 4.0 International (CC BY-NC-ND 4.0) License. 